\title{
A Lower Bound on Navigation Error for Marine Robots Guided by Ocean Circulation Models
}

\author{
Klementyna Szwaykowska and Fumin Zhang
}

\begin{abstract}
This paper establishes the method of controlled Lagrangian particle tracking (CLPT) to analyse the offsets between physical positions of marine robots in the ocean and simulated positions of controlled particles in an ocean model. This offset (which we term CLPT error) has characteristics that are not previously seen in free-drifting ocean sampling platforms with no active control. CLPT error growth over time is exponential until it reaches a turning point that depends only on the resolution of the ocean model, after which the error growth is bounded by polynomial functions of time. In the ideal case, a theoretical lower bound on the steady-state CLPT error can be derived. These characteristics are proved theoretically for particles moving in a planar flow field. The method of CLPT may be applied to improve the accuracy of ocean circulation models and navigation performance of marine robots.
\end{abstract}

\section{INTRODUCTION}

Ocean circulation modeling is an area of active research in physical oceanography. One established method for improving the accuracy of ocean circulation models is through use of Lagrangian particle tracking (LPT). Under this approach, trajectories of GPS-tracked drifting platforms advected freely by ocean flows are compared with numerically modelled trajectories obtained using the ocean circulation model. Position data collected from free-drifting platforms can be assimilated into the circulation models, improving the longterm accuracy of predicted flow values in certain cases [6], [9], [12], [13]. The offset between the physical positions of the floats and their simulated counterparts provides a measure for the accuracy of ocean models. However, this method is sensitive to the deployment positions of the floats, and depending on flow conditions, the offset between the true and simulated positions can grow exponentially over time.

In recent years, autonomous underwater vehicles (AUVs) have been developed as small, inexpensive, and flexible controlled platforms for oceanographic studies (see ex. [2], [16]). AUVs have many of the same advantages as Lagrangian floats, such as low deployment cost and easy scalability of the number of deployed platforms, and may be used to replace floats in certain applications [11], [17]. Unlike floats, AUVs have controlled velocity inputs, and can make real-time adjustments to mission goals, such as increasing sampling density in a particular region of interest,

School of Electrical and Computer Engineering, Georgia Institute of Technology. Email: klimka,fumin@ece.gatech.edu

The research work is supported by ONR grants N00014-08-1-1007, N00014-09-1-1074, and N00014-10-10712 (YIP), and NSF grants ECCS0841195 (CAREER), CNS-0931576, and ECCS-1056253. We would like to thanks the HOPS team, the NRL NCOM team and the JPL ROMS team for providing ocean models. or navigating to track a particular phenomenon. Navigation of autonomous vehicles in unstructured ocean environments, however, presents a significant challenge, and requires some knowledge of the underlying flow; at the same time, there has been interest among physical oceanographers in using fleets of AUVs to help improve ocean circulations models, as has been done previously with floats.

The use of ocean model data for AUV navigation has been demonstrated in a number of research efforts. The Adapting Sampling and Prediction (ASAP) experiment [1], [8], a fleet of AUVs was used for cooperative sampling of a domain over the course of 6 weeks. AUV trajectories were generated based on flow predictions from ocean models, and adapted to changing flow conditions [7], [8], [10], [18]. A series of algorithms for ocean-model based control of an AUV to track plumes in the ocean, is demonstrated in [14]; in this case, the data collected by the AUVs were fed back to the ocean model to improve accuracy of future flow forecasts. A scheme for incorporating data assimilation goals into AUV trajectory planning is proposed in [5], where the AUV's path is chosen to minimize the a posteriori error covariance of the modelled flow after data assimilation.

Autonomous underwater vehicles may prove to be an important asset in the continued development of ever more accurate ocean models. We envision possibilities of realtime integration of localization and navigation performance of underwater vehicles with ocean modeling, to improve both the navigation performance of the AUVs, and the accuracy of ocean model flow predictions. In this paper, we talk about a new method of controlled Lagrangian particle tracking (CLPT), which creates a natural framework for understanding the interaction between flows estimated or predicted by ocean models and controlled particle trajectories. CLPT quantifies the offset between predicted and actual positions of particles under active feedback control moving in ocean flows, with guidance from ocean circulation models.

In this paper we theoretically justify the unique characteristics of the error growth associated with the CLPT that have not been previously observed in LPT. We show that the CLPT error typically grows exponentially for a relatively short period of time, then will grow much slower with a rate bounded above by polynomial functions of time. The size of error at the turning point depends only on the resolution of the ocean model. In contrast, the error growth for LPT tends to be exponential most of the time. Our results show that CLPT error grows much slower than LPT error, and hence may be more useful for assimilation of data into the ocean model. Our theoretical results are supported by data collected 
during a field experiment.

The rest of this paper is organized as follows. The method of the controlled Lagrangian particle tracking and CLPT error is introduced in Section II. The CLPT error bound is calculated in Section III for particles moving in a planar flow field. The results are compared with observations made during the Adaptive Sampling and Prediction (ASAP) experiment in Monterey Bay in 2006 (Section IV).

\section{Controlled Lagrangian Particle Tracking}

Controlled Lagrangian particle tracking is used to model the motions of vehicles with controlled velocity inputs in ocean flow fields. Each vehicle is modelled as a massless particle whose velocity is the sum of ambient flow velocity $\mathcal{F}$ and control velocity input $\mathbf{u}$. In this paper we consider the horizontal motion of the particles. Particle position at time $t$ is given by $\mathbf{x}(t) \in \mathbb{R}^{2}$, obtained by integrating the following equation:

$$
\mathrm{d} \mathbf{x}(t)=(\mathcal{F}(\mathbf{x}, t)+\mathbf{u}(\mathbf{x}, t)) \mathrm{d} t
$$

Realistic trajectories for particles drifting in a flow field can be obtained by decomposing $\mathcal{F}$ into two constituent components: a mean flow $\mathbf{F}$, and a stochastic eddy diffusion field $\nu$, which includes small-scale flow fluctuations due to physical eddies, as well as waves, wind-driven flows, and other turbulent components. It has been shown that this approach can be used effectively to model trajectories of passive drifter platforms in the ocean, provided that the parameters describing the turbulent flow components are known or can be estimated [3], [4]. A good first-order model of particle trajectories can be given by a Markovian randomwalk model [4], described using the following modified Langevin equation:

$$
\begin{aligned}
& \mathrm{d} \mathbf{x}=(\mathbf{F}(\mathbf{x}, t)+\nu+\mathbf{u}(\mathbf{x}, t)) \mathrm{d} t \\
& \mathrm{~d} \nu=-A \nu \mathrm{d} t+\Lambda \mathrm{d} \omega
\end{aligned}
$$

where $\mathbf{F}(\mathbf{x}, t)$ is the underlying mean flow, and $\nu$ is the eddy diffusion term. The matrices $A$ and $\Lambda$ are given by

$$
A=\left[\begin{array}{cc}
\frac{1}{\tau} & \Omega \\
-\Omega & \frac{1}{\tau}
\end{array}\right] \quad \Lambda=\left[\begin{array}{cc}
\sigma \sqrt{\frac{2}{\tau}} & 0 \\
0 & \sigma \sqrt{\frac{2}{\tau}}
\end{array}\right]
$$

where $\sigma$ is the variance of the stochastic velocity fluctuations, $\tau$ represents Lagrangian correlation time, and $\Omega$ is the spin parameter. The stochastic input $\omega$ represents zero-mean, Gaussian white noise.

In practice, since the stochastic velocity input $\nu$ is unknown, the particle position is estimated using:

$$
\mathrm{d} \mathbf{z}(t)=(\hat{\mathbf{F}}(\mathbf{z}, t)+\mathbf{u}(\mathbf{z}, t)) \mathrm{d} t
$$

where $\mathbf{z}(t)$ is an estimate of the particle's position $\mathbf{x}(t)$, and $\hat{F}$ is the estimated mean flow, obtained from ocean models.

\section{A. CLPT Error}

The more realistic model of particle motion in (2) can be used to gauge the accuracy of position prediction made using the simple model (4). The position prediction error $\mathbf{e}_{\text {pos }} \in \mathbb{R}^{2}$ is defined as:

$$
\mathbf{e}_{\text {pos }}(t)=\mathbf{x}(t)-\mathbf{z}(t) .
$$

We set the initial time $t_{0}=0$, and $\mathbf{z}(0)=\mathbf{x}(0)$, so that $\mathbf{e}(0)=\left[\begin{array}{ll}0 & 0\end{array}\right]^{\mathrm{T}}$. The magnitude of the position prediction error is defined as the CLPT error $e(t)$ :

$$
e(t):=\|\mathbf{x}(t)-\mathbf{z}(t)\|
$$

The CLPT error may be taken as a measure of the performance of the ocean-model based position prediction.

\section{B. CLPT error dynamics}

In the following section, we will show that there is a fundamental limit on the quality of the position prediction estimate that can be obtained using the ocean model. This is done by deriving a lower bound on the expected value of the CLPT error.

The lower bound on the CLPT error can be derived as follows: by Jensen's inequality,

$$
\left\|E\left(\mathbf{e}_{\text {pos }}(t)\right)\right\| \leq E\left(\left\|\mathbf{e}_{\text {pos }}(t)\right\|\right)=E(e(t)) ;
$$

thus, $\left\|E\left(\mathbf{e}_{\text {pos }}(t)\right)\right\|$ gives a lower bound on the expected value of $E(e(t))$. It remains only to find $E\left(\mathbf{e}_{\text {pos }}(t)\right)$.

The growth in the position estimation error over time is given by:

$$
\begin{aligned}
\mathrm{d} \mathbf{e}_{p o s}(t) & =[\mathbf{F}(\mathbf{x}, t)+\mathbf{u}(\mathbf{x}, t)-\hat{\mathbf{F}}(\mathbf{z}, t)-\mathbf{u}(\mathbf{z}, t)+\nu] \mathrm{d} t \\
\mathrm{~d} \nu & =-A \nu \mathrm{d} t+\Lambda \mathrm{d} \omega .
\end{aligned}
$$

The control velocity $\mathbf{u}$ can be designed to cancel the effects of the estimated mean flow. In the remainder of this paper, we will make the following assumption:

(A1) A flow-cancelling controller has been implemented, so that:

$$
\mathbf{u}(\chi, t)=-\hat{\mathbf{F}}(\chi, t)
$$

for $\chi(t)=\mathbf{x}(t)$ or $\mathbf{z}(t)$.

Let $\mathbf{f}(\mathbf{z}, t):=\mathbf{F}(\mathbf{z}, t)-\hat{\mathbf{F}}(\mathbf{z}, t)$ be the error in the estimated mean flow $\hat{\mathbf{F}}$. Under assumption (A1), position estimation error growth may be modelled as:

$$
\begin{aligned}
\mathrm{d} \mathbf{e}_{p o s}(t) & =[\mathbf{f}(\mathbf{x}, t)+\nu] \mathrm{d} t \\
\mathrm{~d} \nu & =-A \nu \mathrm{d} t+\Lambda \mathrm{d} \omega .
\end{aligned}
$$

The expected value of the position estimation error evolves in times as:

$$
\begin{aligned}
\mathrm{d} E\left(\mathbf{e}_{p o s}\right) / \mathrm{d} t & =E(\mathbf{f}(\mathbf{x}, t))+E(\nu) \\
\mathrm{d} E(\nu) / \mathrm{d} t & =-A E(\nu) .
\end{aligned}
$$

Note that the dynamics of $\nu$ are independent of $\mathbf{e}_{\text {pos }}$. The matrix $-A$ is Hurwitz, so that $E(\nu)=0$ is a stable equilibrium of (12). Therefore, as $t \rightarrow \infty, E(\nu)=0$, and 
$E\left(\mathbf{e}_{\text {pos }}\right)$ will converge to stable equilibrium points of the equation

$$
\mathrm{d} E\left(\mathbf{e}_{p o s}\right) / \mathrm{d} t=E(\mathbf{f}(\mathbf{x}, t)) .
$$

In general $\mathbf{f}$ is an unknown function of position and time, whose values depend on the particular realization of the ocean flow and on the model used. Given a particular ocean flow and model pair, however, $\mathbf{f}$ is deterministic, and the conditional expected value $E(\mathbf{f})=\mathbf{f}$.

Given estimated flow cancellation, the expected position of the simulated vehicle remains $E(\mathbf{z}(t))=\mathbf{z}(0)$. Therefore, $E\left(\mathbf{e}_{\text {pos }}(t)\right)=E(\mathbf{x}(t)-\mathbf{z}(0))$. We can assume, without loss of generality, that the vehicle starts at the origin, $\mathbf{x}(0)=$ $\mathbf{z}(0)=\left[\begin{array}{ll}0 & 0\end{array}\right]^{\mathrm{T}}$. Then $E\left(\mathbf{e}_{\text {pos }}(t)\right)$ given by (13) will be equal to $\mathbf{x}(t)$ in the following system:

$$
\mathrm{d} \mathbf{x}=\mathbf{f}(\mathbf{x}, t) \mathrm{d} t, \quad \mathbf{x}(0)=0
$$

where $\mathbf{f}(\mathbf{x}, t):=\hat{\mathbf{F}}(\mathbf{x}, t)-\mathbf{F}(\mathbf{x}, t)$. For simplicity, we will ignore the time variation of $\mathbf{f}$ and treat it as a function of $\mathbf{x}$ only. We also assume that $\mathbf{f}$ has equilibria; that is, there exits a non-empty set $\mathbf{X}$ s.t. $f(\mathbf{x}, t)=0 \forall \mathbf{x} \in \mathbf{X}$.

For a given realization of $\mathbf{f}$, say $\mathbf{x}(t)$ in eq. (14) approaches $\mathbf{x}_{e q}$, a stable equilibrium of (14), as $t \rightarrow \infty$, then $\left\|\mathbf{x}_{e q}-\mathbf{z}(0)\right\|$ is a lower bound for the CLPT error.

\section{Comparison with LPT error}

For freely-drifting agents in the ocean, the position prediction error can be expressed as in eq. (8), with control $\mathbf{u} \equiv 0$ :

$$
\begin{aligned}
\mathrm{d} \mathbf{e}_{p o s}(t) & =[\mathbf{F}(\mathbf{x}, t)-\mathbf{F}(\mathbf{z}, t)] \mathrm{d} t-\mathbf{f}(\mathbf{z}, t) \mathrm{d} t+\nu \mathrm{d} t \\
\mathrm{~d} \nu & =-A \nu \mathrm{d} t+\Lambda \mathrm{d} \omega .
\end{aligned}
$$

The first term in the error growth equation depends on the underlying flow velocity at two different points: the simulated vehicle position $\mathbf{z}(t)$ and the true position $\mathbf{x}(t)$. Given a slight initial offset between $\mathbf{z}$ and $\mathbf{x}$, the error growth due to this term alone (setting $\mathbf{f} \equiv 0$ and $\nu \equiv 0$ ) is:

$$
\mathrm{d} \mathbf{e}_{\text {pos }}(t)=[\mathbf{F}(\mathbf{x}, t)-\mathbf{F}(\mathbf{z}, t)] \mathrm{d} t .
$$

This can be interpreted as the offset between two particles, initially close together, drifting in an ocean flow. Even in simple flow fields, the separation between two such particles may grow exponentially without bound (this fast divergence of trajectories from close initial conditions is termed deterministic chaos) [13]. Nothing can be said about the longterm error growth rate. This is in contrast with the case of controlled particles, where error growth rate will be shown to decrease in the long term.

\section{THEORETICAL LOWER BOUND ON CLPT ERROR}

In this section, we derive a theoretical lower bound on the expected CLPT error for a planar flow field. The lower bound on the expected error is a function of the ocean model gridsize.

We make the following assumptions:

(B1) The ocean model outputs an estimate $\hat{\mathbf{F}}$ of the flow $\mathbf{F}$ at each gridpoint on a uniform square grid over $\mathbb{R}^{2}$ with gridsize $h$, where $\mathbf{x}_{k, l}=[k h, l h]^{\mathrm{T}}$ is a gridpoint indexed by $(k, l)$. We assume that the values of $\mathbf{f}(\mathbf{x})=$ $\hat{\mathbf{F}}(\mathbf{x})-\mathbf{F}(\mathbf{x})$ at the grid points are iid symmetricallydistributed random variables, which will be denoted by $\vec{\xi}_{k, l}=\left[\xi_{k, l}^{1} \xi_{k, l}^{2}\right]^{\mathrm{T}}$ (where $\xi_{k, l}^{1}=f^{1}\left(\mathbf{x}_{k, l}\right)$ and $\xi_{k, l}^{2}=$ $f^{2}\left(\mathbf{x}_{k, l}\right)$ are mutually independent). The pdf of $\xi_{k, l}^{i}$, $i=1,2$, will be denoted by $\rho_{\xi}$.

(B2) The values of $\mathbf{f}$ at any point can be approximated by taking the bilinear interpolation of grid point values

(B3) The physical and simulated particles are initialized at the same position, $\mathbf{z}(0)=\mathbf{x}(0)$.

Given the above, we find a lower bound on the $\ell^{1}$ norm $E\left(\left\|\mathbf{x}_{e q}-\mathbf{x}(0)\right\|_{1}\right)$ as $E\left(\left\|\mathbf{x}_{*}-\mathbf{x}(0)\right\|_{1}\right)$, where $\mathbf{x}_{*}$ is defined as the smallest equilibrium of the flow $\mathbf{f}$ which satisfies the necessary stability condition $\partial \mathbf{f}^{i} / \partial \mathbf{x}_{i}<0$ for $i=1,2$ :

$\mathbf{x}_{*}=\underset{\mathbf{x} \in \mathbb{R}^{2}}{\operatorname{argmin}}\left\{\|\mathbf{x}-\mathbf{x}(0)\|_{1}: \mathbf{f}\left(\mathbf{x}_{*}\right)=0, \partial \mathbf{f}^{i} / \partial \mathbf{x}_{i}(\mathbf{x})<0 \forall i\right\}$

The main contribution of this section is summarized in the following theorem:

Theorem 3.1: The expected value of the lower bound on the 1-norm of the steady-state position error $\left\|\mathbf{x}_{*}\right\|_{1}$ satisfies $E\left(\left\|\mathbf{x}_{*}-\mathbf{x}(0)\right\|_{1}\right) \leq 2 \tilde{E}$, where

$$
\begin{aligned}
& \tilde{E}:= 8 \sum_{k=0}^{\infty}\left\{\frac{(k+1) h^{2}}{4^{k+1}} \int_{-k h}^{-(k+1) h} \int_{0}^{\infty} \frac{v}{x} \zeta(v, x) \mathrm{d} v \mathrm{~d} x\right. \\
&+\frac{k h}{4^{k+1}} \int_{-k h}^{-(k+1) h} \int_{0}^{\infty} \frac{v}{x} \zeta(v, x) \\
&\left.\times\left[1-\int_{0}^{|x|-k h} \int_{0}^{\infty} \frac{4 v}{u^{2}} \zeta(v, u) \mathrm{d} v \mathrm{~d} u\right] \mathrm{d} v \mathrm{~d} x\right\}, \\
& \zeta(v, \cdot):=\rho_{\xi}(v) \rho_{\xi}(v(1-h /(\cdot))),
\end{aligned}
$$

and $h$ is the grid size used by the ocean model. In particular, if $\rho_{\xi}$ is Gaussian, $E\left(\left\|\mathbf{x}_{*}-\mathbf{x}(0)\right\|_{1}\right) \leq 2.52 h$.

In order to prove this theorem, we need the following lemmas. For convenience in notation, we assume that $\mathbf{x}(0)=$ 0 (without loss of generality), and we introduce the following variables:

$$
\begin{gathered}
x_{i}^{+}=\operatorname{argmin}_{x_{i}>0}\left\{\left|x_{i}\right|: f^{i}\left(x_{i} \hat{\mathbf{e}}_{i}\right)=0, \partial f^{i} / \partial x_{i}<0\right\} \\
x_{i}^{-}=\operatorname{argmin}_{x_{i}<0}\left\{\left|x_{i}\right|: f^{i}\left(x_{i} \hat{\mathbf{e}}_{i}\right)=0, \partial f^{i} / \partial x_{i}<0\right\},
\end{gathered}
$$

where $\hat{\mathbf{e}}_{i}$ is the standard $i^{\text {th }}$ basis vector in $\mathbb{R}^{2}$.

Lemma 3.1: The following equations hold:

$$
\begin{array}{r}
\operatorname{Pr}\left(x_{i}^{-} \in(-(k+1) h,-k h) \mid \xi_{0,0}^{i}>0\right)=k / 2^{k+1} \\
\operatorname{Pr}\left(x_{i}^{+}>(k+1) h \mid \xi_{0,0}^{i}>0\right)=1 / 2^{k+1} \\
\operatorname{Pr}\left(x_{i}^{+} \in(k h,(k+1) h) \mid \xi_{0,0}^{i}>0\right)=1 / 2^{k+1} .
\end{array}
$$

Proof: The proof of this lemma is omitted due to space constraints. 
Lemma 3.2: Given $u=x_{i}-x_{k, i}$, the probability distribution

$$
\begin{gathered}
\rho_{\left|x_{*, i}-x_{k, i}\right|}\left(u \mid \xi_{0,0}^{i}>0,\left(x_{*, i}-x_{k, i}\right) \in(0, h)\right)= \\
\frac{4 h}{u^{2}} \int_{0}^{\infty} v \rho_{\xi}(v) \rho_{\xi}(v(1-h / u)) d v U(h / u-1),
\end{gathered}
$$

where $U(\cdot)$ denotes the Heaviside function.

Proof: The proof of this lemma is omitted due to space constraints (see [15]).

Lemma 3.3: The probability that $x_{*, i}$ lies in the interval $(-(k+1) h,-k h)$ is:

$$
\begin{gathered}
\operatorname{Pr}\left(x_{*, i} \in(-(k+1) h,-k h) \mid \xi_{0,0}^{i}>0\right)=\frac{k}{4^{k}} \\
\times\left(\frac{1}{2}-\int_{0}^{\left|x_{i}^{-}\right|-k h} \frac{h}{u^{2}}\left(\int_{0}^{\infty} v \rho_{\xi}(v) \rho_{\xi}(v(1-h / u)) \mathrm{d} v\right) \mathrm{d} u\right) .
\end{gathered}
$$

Proof: See Appendix A.

Lemma 3.4: The probability that $x_{*, i}$ lies in the interval $(k h,(k+1) h)$ is:

$$
\begin{gathered}
\operatorname{Pr}\left(x_{*, i}=x_{i}^{+}, x_{i}^{+} \in(k h,(k+1) h) \mid \xi_{0,0}^{i}>0\right)=\frac{1}{2\left(4^{k}\right)} \\
+\frac{k}{4^{k}}\left(\frac{1}{2}-\int_{0}^{x_{i}^{+}-k h} \frac{h}{u^{2}}\left(\int_{0}^{\infty} v \rho_{\xi}(v) \rho_{\xi}(v(1-h / u)) \mathrm{d} v\right) \mathrm{d} u\right)
\end{gathered}
$$

Proof: See Appendix A.

Lemma 3.5: The value of $E\left(\left|x_{*, i}-x_{i}(0)\right|\right)$ is independent of the initial position $\mathrm{x}(0)$.

Proof: For $x_{i}(0) \in[k h,(k+1) h)$, use a coordinate shift $x \mapsto x^{\prime}=x-k h$ so that $x_{i}^{\prime}(0) \in[0, h)$. Then

$$
\begin{aligned}
& E\left(\left|x_{*, i}-x_{i}(0)\right|\right) \\
& =E\left(x_{*, i}^{\prime}-x_{i}^{\prime}(0) \mid x_{*, i}^{\prime}=x_{i}^{+}>0\right) \operatorname{Pr}\left(x_{*, i}^{\prime}=x_{i}^{+}\right) \\
& \quad+E\left(x_{*, i}^{\prime}+x_{i}^{\prime}(0) \mid x_{*, i}^{\prime}=x_{i}^{-}<0\right) \operatorname{Pr}\left(x_{*, i}^{\prime}=x_{i}^{-}\right) \\
& =E\left(x_{*, i}^{\prime}\right)-\frac{1}{2} x_{i}^{\prime}(0)+\frac{1}{2} x_{i}^{\prime}(0)=E\left(x_{*, i}^{\prime}\right),
\end{aligned}
$$

which is independent of $\mathbf{x}(0)$.

Having these Lemmas, we can now proceed to the proof of Theorem 3.1:

Proof: $\quad$ The $\ell^{1}$ norm of $\mathbf{x}_{*}$ is $\left|x_{*, 1}\right|+\left|x_{*, 2}\right|$. By assumption, the flows along the $\mathrm{x}$ - and $\mathrm{y}$-axes are mutually independent. We proceed by computing the expected value of $\left|x_{*, i}\right|$, for $i=1,2$, along each dimension independently.

By Lemma 3.5 , the value of $E\left(\left|x_{*, i}-x_{i}(0)\right|\right)$ is independent of the initial position $\mathbf{x}(0)$; we therefore assume, without loss of generality, that $\mathbf{x}(0)=\mathbf{z}(0)=0$.

Consider the motion along one axis. Let $\xi_{k}^{i}$, for $i=1,2$, denote the the values of $\xi_{i, j}^{i}$ along the $i^{t h}$ axis; so that $\xi_{k}^{1}=$ $\xi_{k, 0}^{1}$ and $\xi_{k}^{2}=\xi_{0, k}^{2}$. Conditioning on $\xi_{0}^{i}$, we can write:

$$
\begin{aligned}
E\left(\left|x_{*, i}\right|\right) & =\frac{1}{2} \int_{-\infty}^{\infty}\left|x_{i}\right| \rho_{\left|x_{*, i}\right|}\left(x_{i} \mid \xi_{0}^{i}>0\right) \mathrm{d} x_{i} \\
& +\frac{1}{2} \int_{-\infty}^{\infty}\left|x_{i}\right| \rho_{\left|x_{*, i}\right|}\left(x_{i} \mid \xi_{0}^{i}<0\right) \mathrm{d} x_{i} .
\end{aligned}
$$

It follows from the symmetry of $\rho_{\xi}$ that $\int_{-\infty}^{\infty}\left|x_{i}\right| \rho_{\left|x_{*, i}\right|}\left(x_{i} \mid \xi_{0}^{i}>0\right) \mathrm{d} x_{i}=\int_{-\infty}^{\infty}\left|x_{i}\right| \rho_{\left|x_{*, i}\right|}\left(x_{i} \mid \xi_{0}^{i}<\right.$ $0) \mathrm{d} x_{i}$, so (22) can be written:

$$
E\left(\left|x_{*, i}\right|\right)=\int_{-\infty}^{\infty}\left|x_{i}\right| \rho_{\left|x_{*, i}\right|}\left(x_{i} \mid \xi_{0}^{i}>0\right) \mathrm{d} x_{i} .
$$

Note that $x_{*, i}=\operatorname{argmin}_{x_{i}^{+}, x_{i}^{-}}\{|x|\}$, therefore $E\left(\left|x_{*, i}\right|\right)$ can be written as:

$$
\begin{aligned}
& E\left(\left|x_{*, i}\right|\right)= \\
& =\sum_{k=0}^{\infty} \int_{-k h}^{-(k+1) h} x_{i} \rho_{\left|x_{*, i}\right|}\left(x_{i} \mid \xi_{0}^{i}>0, \mathrm{~d} 1\right) \operatorname{Pr}\left(\mathrm{d} 1 \mid \xi_{0}^{i}>0\right) \mathrm{d} x_{i} \\
& +\sum_{k=0}^{\infty} \int_{x_{k, i}}^{x_{k, i}+h} x_{i} \rho_{\left|x_{*, i}\right|}\left(x_{i} \mid \xi_{0}^{i}>0, \mathrm{~d} 2\right) \operatorname{Pr}\left(\mathrm{d} 2 \mid \xi_{0}^{i}>0\right) \mathrm{d} x_{i}
\end{aligned}
$$

where we have adopted the shorthand $\mathrm{d} 1$ for the condition " $x_{*}=x_{i}^{-}, x_{i}^{-} \in(-(k+1) h,-k h)$ " and $\mathrm{d} 2$ for " $x_{*}=$ $x_{i}^{+}, x_{i}^{+} \in(k h,(k+1) h)$ ".

Using Lemmas 3.3 and 3.4, and applying change of variables $u=x_{i}-\left\lfloor\frac{x_{i}}{h}\right\rfloor h, v=\xi_{\left\lfloor\frac{x_{i}}{h}\right\rfloor}^{i}$, we can write (24) as:

$$
\begin{aligned}
& E\left(\left|x_{*, i}\right|\right)=\sum_{k=0}^{\infty} \frac{k}{4^{k+1}}\left\{\int_{-k h}^{-(k+1) h} \psi_{1}\left(x_{i}\right) \mathrm{d} x_{i}\right. \\
& +\int_{-k h}^{-(k+1) h} \psi_{1}\left(x_{i}\right) J\left(x_{i}\right) \mathrm{d} x_{i}+\frac{k+2}{k} \int_{k h}^{(k+1) h} \psi_{2}\left(x_{i}\right) \mathrm{d} x_{i} \\
& \left.+\int_{k h}^{(k+1) h} \psi_{2}\left(x_{i}\right) J\left(x_{i}\right) \mathrm{d} x_{i}\right\}
\end{aligned}
$$

where

$$
\begin{aligned}
& \psi_{1}\left(x_{i}\right):=x_{i} \rho_{\left|x_{*, i}\right|}\left(x_{i} \mid \xi_{0}^{i}>0, \mathrm{~d} 1\right) \\
& \psi_{2}\left(x_{i}\right):=x_{i} \rho_{\left|x_{*, i}\right|}\left(x_{i} \mid \xi_{0}^{i}>0, \mathrm{~d} 2\right) \\
& J\left(x_{i}\right):=1-\int_{0}^{\left|x_{i}\right|-k h^{2}} \frac{4 h}{u^{2}}\left(\int_{0}^{\infty} v \rho_{\xi}(v) \rho_{\xi}\left(v\left(1-\frac{h}{u}\right)\right) \mathrm{d} v\right) \mathrm{d} u .
\end{aligned}
$$

Using the symmetry of $\rho_{\xi}$, we can combine terms in eq. (25) to obtain:

$$
\begin{aligned}
E\left(\left|x_{*, i}\right|\right)= & \sum_{k=0}^{\infty}\left\{\frac{2(k+1) h}{4^{k+1}} \int_{-k h}^{-(k+1) h} \psi_{1}\left(x_{i}\right) \mathrm{d} x_{i}\right. \\
& \left.+\frac{2 k}{4^{k+1}} \int_{-k h}^{-(k+1) h} \psi_{1}\left(x_{i}\right) J\left(x_{i}\right) \mathrm{d} x_{i}\right\}
\end{aligned}
$$

Substituting in the pdf in Lemma 3.2 into $\psi_{1}\left(x_{i}\right)$, we get $E\left(\left|x_{*, i}\right|\right)=\tilde{E}$, where $\tilde{E}$ is given by eq. (17). Then $E\left(\left|\mathbf{x}_{*}\right|_{1}\right)=E\left(\left|x_{*}^{1}\right|+\left|x_{*}^{2}\right|\right)=2 \tilde{E}$. This proves Theorem 3.1 in the general case. If $\xi$ has a Gaussian distribution, the above expression may be evaluated numerically to give $E\left(\left|x_{*, i}\right|\right)=1.26 h$, from which it follows that in this case, $E\left(\left|\mathbf{x}_{*}\right|\right)=2.52 h$. This completes the proof of Theorem 3.1.

We have shown the bound on the $\ell^{1}$ norm of the position estimation error in the 2-D case. This gives a range on the bound of the $\ell^{2}$ error, since $\|\chi\|_{1} / \sqrt{2} \leq\|\chi\|_{2} \leq\|\chi\|_{1}$ for all $\chi \in \mathbb{R}^{2}$. Therefore we can say that in the case that 
$\xi$ are Gaussian random variables, the lower bound on the $\ell^{2}$ norm of the position estimation error satisfies:

$$
E\left(\left\|\mathbf{x}_{*}\right\|_{2}\right) \in[1.78 h, 2.52 h] .
$$

Note that the above calculations do not depend on the strength of the error flow values at the gridpoints, only on the fact that they are independent, identically distributed random variables. The strength (that is, the variance) of the flow, however, does affect the time it takes for the error growth to reach an equilibrium. Since the velocity of the particle in the ocean flow is given by a bilinear interpolation of the flow values at the gridpoints, the higher the variance of the gridpoint values, the higher the mean velocity; and consequently, the faster the particles converge to an equilibrium of the error flow field.

The above approach does not address the coupling between the $\mathrm{x}$ and $\mathrm{y}$-position of the expected equilibrium due to the bilinear interpolation of the gridpoint flow values. Because it is a bilinear interpolation of gridpoint values, the error flow along each of the $\mathrm{x}$ and $\mathrm{y}$ directions is a $\mathcal{C}^{0}$ function in $\mathbb{R}^{2}$; thus, its zero-level sets $\mathbf{f}^{1}(\mathbf{x})=0$ and $\mathbf{f}^{2}(\mathbf{x})=0$ are continuous curves (see Figure 1$)$. The average distance to the nearest equilibrium, therefore, depends not only on the average distance to an equilibrium along a particular axis, but also on the shape of the zero-level set curve. This can be understood via the following simple example: consider two lines $y=0$ and $y=h$, with zerocrossings at $x=1$ and $x=-2$, respectively. The sample mean of the norms of the crossing values is $(|1|+|-2|) / 2=$ 1.5. Consider a straight line joining the two crossing points. The mean distance from the y-axis to this line is $5 / 6 h$. In general, the distance to the zero level set curve along the $\mathrm{x}$ and $\mathrm{y}$-directions is less than the expected distance to an equilibrium along a particular line parallel that axis; for this reason, Theorem 3.1 gives an upper bound on the true value of $E\left(\left\|\mathbf{x}_{*}\right\|_{1}\right)$ at steady state.

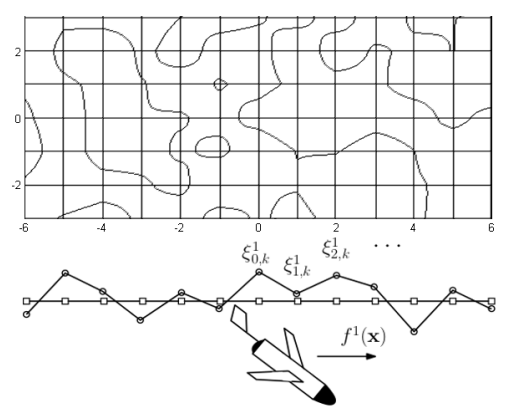

Fig. 1. Zero level set of the error flow in the $x$-direction, $\mathbf{f}^{1}(\mathbf{x})=0$ and cross-section of $\mathrm{x}$-direction flow at constant value of $x_{2}$.

\section{THE ASAP FIELD EXPERIMENT}

In deriving the lower bound on the magnitude of the position estimation error between simulated particles and particles moving in real ocean environments, we have made several simplifying assumptions about the structure of the error field. To demonstrate that the results hold in more complicated situations, we compare them with simulation and observations made during the 2006 Adaptive Sampling and Prediction (ASAP) field experiment in Monterey Bay, CA, in August 2006. The purpose of the experiment was to study the three-dimensional heat and mass transfer dynamics of the coastal upwelling center in the Bay [8]. A $22 \times 40 \mathrm{~km}$ control volume was monitored for a period of one month using a heterogeneous ocean sampling network, which included a fleet of underwater gliders. The gliders moved along predetermined tracks and were coordinated through a centralized onshore controller running the Glider Coordinated Control System (GCCS) (see [10]).

A virtual glider experiment was run alongside the physical one, with gliders in the virtual fleet running the same onboard control laws and initialized at the same positions as their physical counterparts. Their motions were simulated in GCCS, using flow data from an ocean model. The experiment was repeated for three different ocean models: the Navy Coastal Ocean Model (NCOM), the Harvard Ocean Prediction System (HOPS), and the Regional Ocean Modelling System (ROMS) developed by NASA JPL. Each virtual experiment was run over a period of 24 hours.

A plot of the norm of the error in the position prediction over time (averaged over all gliders and all days for which data is available) obtained using the virtual glider model is shown in Figure 2. Note that the error grows approximately exponentially until a value of about twice the gridsize is reached; past this point, the growth rate slows down. This agrees with the CLPT prediction of lower bound on the error, and with the contribution of the stochastic eddy diffusivity term in the Langevin equation.

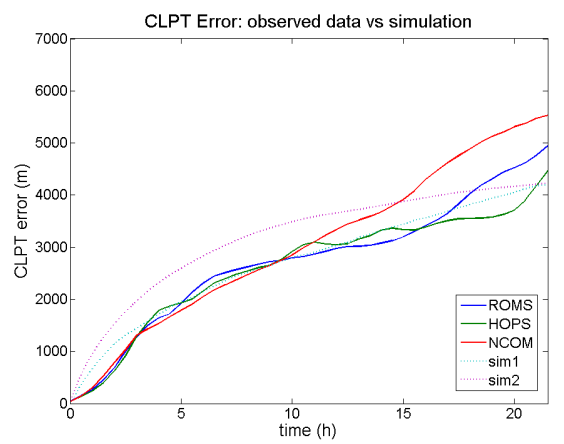

Fig. 2. CLPT error growth observed in the ASAP experiment, averaged over all gliders and all days of collected data. Plots for each of the ocean models used are shown. Two plots of CLPT error simulated using the Langevin equation to model movement of the physical glider in the ocean. Sim1 corresponds to a simulation with $\sigma=0.32$, Lagrangian correlation time $\tau=0.28 \mathrm{hrs}$., and $\sigma_{\xi}=0.1$. Sim2 corresponds to a simulation with $\sigma=0.05, \tau=0.28 \mathrm{hrs}$., and $\sigma_{\xi}=0.3$. The simulations were run with gridsize of $2.2 \times 1.8 \mathrm{~km}$, the gridsize used by ROMS in the ASAP experiment. The simulated CLPT error growth results give good qualitative agreement with the observed experimental data.

\section{CONCLUSION AND FUTURE WORK}

This paper develops the theory of controlled Lagrangian particle tracking (CLPT). In particular, we theoretically justified the observations made through experiments that the 
CLPT error has slower growth rate than the LPT error. We have discovered that the CLPT error may approach a theoretical lower bound that is determined by the gridsize of the ocean model that is used for making position predictions.

In our treatment of the CLPT error, we have assumed a particularly simple structure for the error flow in order to make our calculations tractable. This error flow is, in reality, very difficult to characterize. In future work, we propose to incorporate observations of glider position (made at surfacing times) to update the model flow and to create a modelbased observer for the positions of actuated agents. This approach will be used to obtain more accurate positioning of the underwater vehicles, as well as to study the true structure of the error flow.

\section{APPENDIX A}

This appendix contains a sketch of the proofs of lemmas 3.3 and 3.4 in III.

In Lemma 3.3, $\operatorname{Pr}\left(x_{*, i} \in(-(k+1) h,-k h) \mid \xi_{0,0}^{i}>0\right)$ is given by:

$$
\begin{aligned}
& \operatorname{Pr}\left(x_{*}=x_{i}^{-}, x_{i}^{-} \in(-(k+1) h,-k h) \mid \xi_{0,0}^{i}>0\right)= \\
& \operatorname{Pr}\left(\left|x_{i}^{-}\right|<\left|x_{i}^{+}\right|, x_{i}^{-} \in(-(k+1) h,-k h) \mid \xi_{0,0}^{i}>0\right) .
\end{aligned}
$$

We will avoid explicitly writing the dependence on $\xi_{0,0}^{i}$ in future equations to avoid notational clutter. The variables $x_{i}^{-}$ and $x_{i}^{+}$are mutually independent, so that

$$
\begin{aligned}
& \operatorname{Pr}\left(\left|x_{i}^{-}\right|<\left|x_{i}^{+}\right|, x_{i}^{-} \in(-(k+1) h,-k h)\right)= \\
& \operatorname{Pr}\left(x_{i}^{-} \in(-(k+1) h,-k h)\right) \operatorname{Pr}\left(x_{i}^{+}>(k+1) h\right)+ \\
& \operatorname{Pr}\left(x_{i}^{-} \in(-(k+1) h,-k h)\right) \operatorname{Pr}\left(x_{i}^{+} \in\left(\left|x_{i}^{-}\right|,(k+1) h\right)\right) .
\end{aligned}
$$

where

$$
\begin{aligned}
& \operatorname{Pr}\left(x_{i}^{+} \in\left(\left|x_{i}^{-}\right|,(k+1) h\right)\right)=\operatorname{Pr}\left(x_{i}^{+} \in(k h,(k+1) h)\right) \\
& \times \operatorname{Pr}\left(x_{i}^{+} \in\left(\left|x_{i}^{-}\right|,(k+1) h\right) \mid x_{i}^{+} \in(k h,(k+1) h)\right) .
\end{aligned}
$$

Then, using Lemma 3.1, eq. (29) can be written as:

$$
\begin{gathered}
\operatorname{Pr}\left(\left|x_{i}^{-}\right|<\left|x_{i}^{+}\right|, x_{i}^{-} \in(-(k+1) h,-k h)\right)=\frac{k}{4^{k+1}} \\
\times\left(1+\operatorname{Pr}\left(x_{i}^{+} \in\left(\left|x_{i}^{-}\right|,(k+1) h\right) \mid x_{i}^{+} \in(k h,(k+1) h)\right)\right) .
\end{gathered}
$$

The remaining expression, $\operatorname{Pr}\left(x_{i}^{+} \in\left(\left|x_{i}^{-}\right|,(k+1) h\right) \mid x_{i}^{+} \in\right.$ $(k h,(k+1) h))$, may be calculated as follows:

$$
\begin{aligned}
& \operatorname{Pr}\left(x_{i}^{+} \in\left(\left|x_{i}^{-}\right|,(k+1) h\right) \mid x_{i}^{+} \in(k h,(k+1) h)\right) \\
& =1-\operatorname{Pr}\left(x_{i}^{+} \in\left(k h,\left|x_{i}^{-}\right|\right) \mid x_{i}^{+} \in(k h,(k+1) h)\right) \\
& =1-\int_{k h}^{\left|x_{i}^{-}\right|} \rho_{x_{i}^{-}}(x) \mathrm{d} x,
\end{aligned}
$$

where $x_{i}^{-} \in(k h,(k+1) h)$. Using the change of variables $u=x_{i}-k h, v=\xi_{k, 0}^{i}$ and the distribution for $u_{*}=x_{*, i}-$ $x_{k, i}=x_{i}^{+}-k h$ in Lemma 3.2, we have:

$$
\begin{aligned}
& \operatorname{Pr}\left(x_{i}^{+} \in\left(\left|x_{i}^{-}\right|,(k+1) h\right) \mid x_{i}^{+} \in(k h,(k+1) h)\right) \\
& =1-\int_{0}^{\left|x_{i}^{-}\right|-k h} \frac{4 h}{u^{2}}\left(\int_{0}^{\infty} v \rho_{\xi}(v) \rho_{\xi}(v(1-h / u)) \mathrm{d} v\right) \mathrm{d} u .
\end{aligned}
$$

Plugging this expression into eq. (31) gives eq. (20). This completes the proof. Lemma 3.4 can be proved in an analogous way; we omit the details of the proof here due to space constraints.

\section{REFERENCES}

[1] Asap: Adaptive sampling and prediction. Online. http://www.princeton.edu/ dcsl/asap/.

[2] C.C. Eriksen, T.J. Osse, R.D. Light, T. Wen, T.W. Lehman, P.L. Sabin, J.W. Ballard, and A.M. Chidoi. Seaglider: a long-range autonomous underwater vehicle for oceanographic research. IEEE Journal of Ocean Engineering, 26(4):424-436, 2001.

[3] P. Falco, A. Griffa, P.M. Poulain, and E. Zambianchi. Transport properties in the adriatic sea as deduced from drifter data. J. Phys. Oceanography, 30:2055-2071, 2000.

[4] A.C. Haza, L.I. Piterbarg, P. Martin, T.M. Ozgokmen, and A. Griffa. A lagrangian subgridscale model for particle transport improvement and application in the adriatic sea using the navy coastal ocean model. Ocean Modeling, 17:68-91, 2007.

[5] F.S. Hover. Path planning for data assimilation in mobile environmental monitoring systems. In Proc. IEEE/RSJ Int. Conf. on Intelligent Robots and Systems, pages 213-218, St. Luis, MO, 2009.

[6] L. Kuznetsov, K. Ide, and C.K.R.T. Jones. A method for assimilation of lagrangian data. Monthly Weather Review, 131(10):2247-2260, 2003.

[7] N.E. Leonard, D. Paley, F. Lekien, R. Sepulchre, D. Fratantoni, and R. Davis. Collective motion, sensor networks, and ocean sampling. Proc. of the IEEE, special issue on the emerging technology of networked control systems, 95:48-74, 2007.

[8] N.E. Leonard, D.A. Paley, R.E. Davis, D.M. Fratantoni, F. Lekien, and F. Zhang. Coordinated control of an underwater glider fleet in an adaptive ocean sampling field experiment in monterey bay. Journal of Field Robotics, Special Issue on State of the Art in Maritime Autonomous Surface and Underwater Vehicles, 27(6):718-740, 2010.

[9] T.M. Özgökmen, A. Griffa, A.J. Mariano, and L.I. Piterbarg. On the predictability of lagrangian trajectories in the ocean. Journal of Atmospheric and Oceanic Technology, 1(3):366-383, 2000.

[10] D.A. Paley, F. Zhang, D.M. Fratantoni, and N.E. Leonard. Cooperative control systems for ocean sampling: The glider coordinated control system. IEEE Transaction on Control System Technology, 16(4):735744, 2008.

[11] D.L. Rudnick, R.E. Davis, C.C. Eriksen, D.M. Fratantoni, and M.J. Perry. Underwater gliders for ocean research. Journal of the Marine Technology Society, 38:48-59, 2004.

[12] H. Salman, K. Ide, and C.K.R.T. Jones. Using flow geometry for drifter deployment in lagrangian data assimilation. Tellus, 60A:321$335,2008$.

[13] H. Salman, L. Kuznetsov, C.K.R.T. Jones, and K. Ide. A method for assimilating lagrangian data into a shallow-water-equation ocean model. Monthly Weather Review, 134(4):1081-1101, 2006.

[14] R.N. Smith, Y. Chao, P.P. Li, D.A. Caron, B.H. Jones, and G.S Sukhatme. Planning and implementing trajectories for autonomous underwater vehicles to track evolving ocean processes based on predictions from a regional ocean model. International Journal of Robotics Research, 26(12), 2010.

[15] K. Szwaykowska and F. Zhang. A lower bound for controlled lagrangian particle tracking error. In Proc. IEEE Conf. on Decision and Control, 2010.

[16] D.C. Webb, P.J. Simonetti, and C.P. Jones. Slocum: an underwater glider propelled by environmental energy. IEEE Journal of Oceanic Engineering, 26(4):447-452, 2001.

[17] L.L. Whitcomb, D.R. Yoerger, H. Singh, and J. Howland. Advances in underwater robot vehicles for deep ocean exploration: Navigation, control, and survey operations. In Robotics Research Ninth International Symposium, pages 439-448, London, 2000.

[18] F. Zhang, D. M. Fratentoni, D. Paley, N. E. Leonard, and J. M. Lund. Control of coordinated patterns for ocean sampling. International Journal of Control, Special Issue on Navigation, Guidance and Control of Uninhabited Underwater Vehicles, 80(7):1186-1199, 2007. 